\title{
Laboratory-based X-ray NanoCT Explores Morphology of a Zebrafish Embryo
}

$\underline{\text { Simone Ferst }}^{1,2, *}$, Brian Metscher ${ }^{3}$, Mark Müller ${ }^{1,2}$, Sebastian Allner ${ }^{1,2}$, Martin Dierolf ${ }^{1,2}$, Madleen Busse $^{1,2}$, Klaus Achterhold ${ }^{1,2}$, Bernhard Gleich ${ }^{2}$ and Franz Pfeiffer ${ }^{1,2}$

1. Department of Physics, Technical University of Munich, Garching, Germany

2. Munich School of Bioengineering, Technical University of Munich, Garching, Germany

3. Department of Theoretical Biology, University of Vienna, Vienna, Austria

* Corresponding author, simone.ferstl@tum.de

In recent years, the field of microCT imaging has evolved, striving for higher resolutions below the micrometre range. At synchrotron facilities, the high brilliance of the radiation already allows for resolutions in the nanometre range [1], while laboratory-based setups have used different approaches to resolve structures smaller than one micrometre. One of the two main approaches is to use X-ray optics, such as Fresnel zone plates, combined with high flux sources. Thereby resolutions below $100 \mathrm{~nm}$ are accomplished [2]. Another technique is based on the combination of mere geometrical magnification and an X-ray source featuring small X-ray focal spots. Until recently, this approach was limited to a maximum resolution of about $400 \mathrm{~nm}$ [3]. Despite the lower resolving power, setups using solely geometrical magnification have proven themselves valuable since they allow larger field of views (FOVs), shorter acquisition times and work with broad energy spectra. We present an innovative tabletop setup, which is based on geometrical magnification and comprises of a nano-focus X-ray source and a photon counting detector. The setup achieves routinely resolutions down to $100 \mathrm{~nm}$ [4]. This allows non-destructive imaging of inner structures in 3D with very high resolution $[4,5]$. To explore the potential of our nanoCT setup for the field of biology, we imaged a Zebrafish embryo 48 hours post fertilization. Our comparative analyses show that the nanoCT can resolve the surface morphology of the fish embryo at a similar level of detail as scanning electron microscopy (SEM) and reproduce the internal features of the specimen.

The new setup consists of a nano-focus X-ray source, an overhead rotation stage and a photon counting detector. The nano-focus X-ray tube (Excillum AB, Sweden) provides full control of the spot size. Advanced electron optics in combination with a thin tungsten transmission target enables the source to reach focal spots with FWHM values down to $200 \mathrm{~nm}$ in its current state. The X-ray camera is a PILATUS 300K-W detector with a $1.0 \mathrm{~mm}$ thick silicon sensor with an image area of $1475 \mathrm{x} 195$ square pixels with a side length of $172 \mu \mathrm{m}$. It is operated in single-photon counting mode, which allows signal acquisition without readout or dark current noise. Thereby, it ensures good image quality even with low $\mathrm{X}$-ray flux [6]. The FOV is given as $1400 \mathrm{x}$ voxel size in horizontal direction and $190 \mathrm{x}$ voxel size in the vertical direction. The FOV can be extended vertically by combining several CT scans.

For this study, we measured a $2 \mathrm{~mm}$ long and $400 \mu \mathrm{m}$ thick embryo of a Zebrafish 48 hours post fertilization. Prior to analyses the formalin-fixed sample was stained with phosphotungstic acid and mounted in agarose [7]. 18 datasets were acquired and subsequently combined vertically to the presented volume data. Each dataset of 1599 projections was measured over $360^{\circ}$ at an exposure time of $1 \mathrm{~s}$ with an effective voxel size of $800 \mathrm{~nm}$. This results in a total acquisition time of 20 hours. The source was operated at a peak voltage of $60 \mathrm{kV}$. The sample was reconstructed using a filtered backprojection algorithm. 

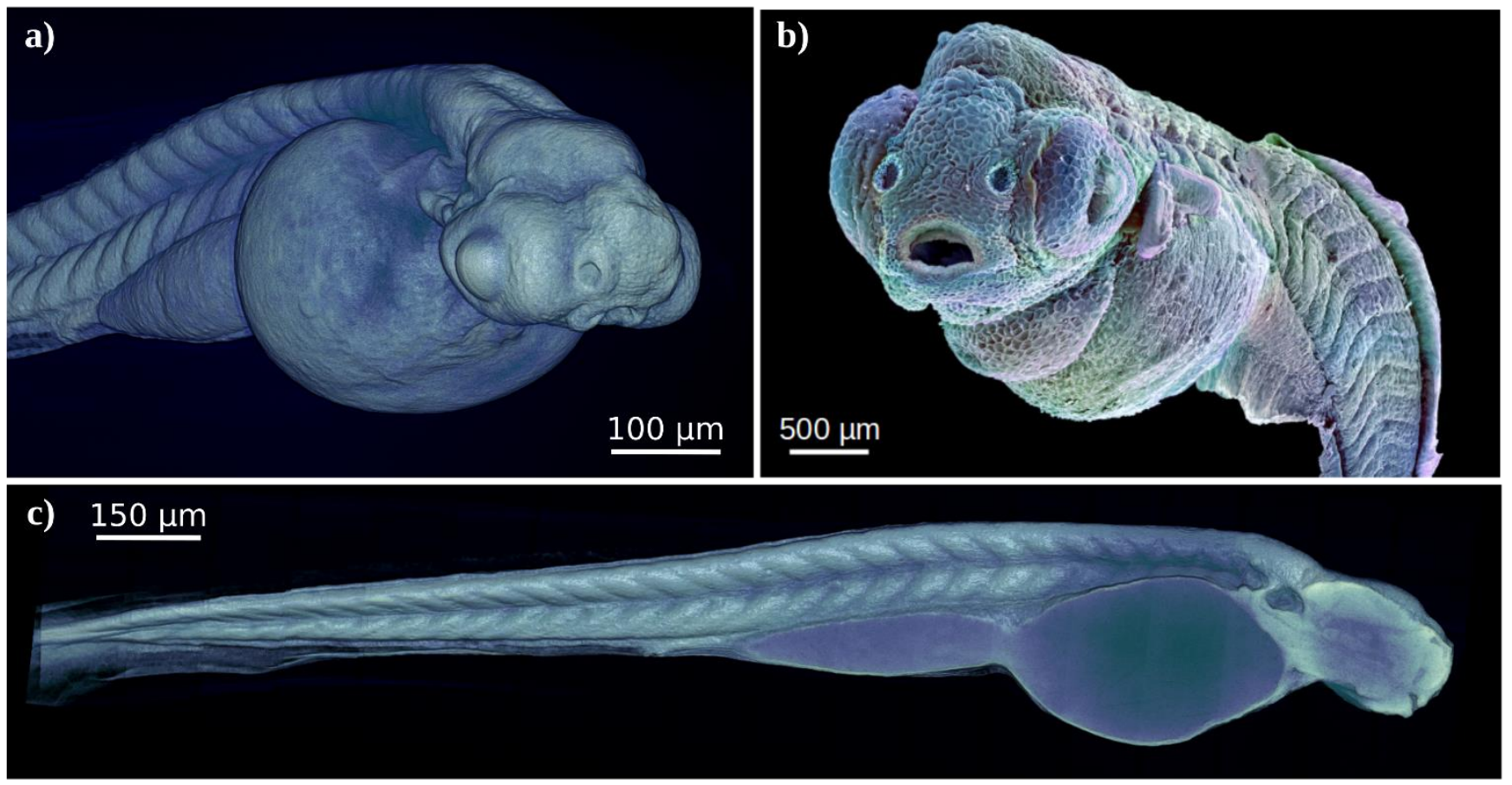

Figure. 1. a) NanoCT volume rendering of a zebrafish embryo 48 hours post fertilization. b) Falsecoloured scanning electron micrograph of a zebrafish embryo 4 days post fertilization, adapted from [8]. c) Digital section through the volume rendering of the specimen.

The resulting nanoCT data were then compared with SEM data obtained from a 4 day old zebrafish embryo with an approximate length of $1 \mathrm{~cm}$ (Figure 1a-b) [8]. The nanoCT data depicts similar surface features as the SEM data (Figure 1a-b). Furthermore, the nanoCT data set provides information about the inner structures of the sample (Figure 1c). Especially throughout the head region various features are visible. Thereby, it is possible to track anatomical components within a biological sample in $3 \mathrm{D}$, which allows to derive hitherto unknown information about the relations and functioning of mechanisms within a specimen [4].

\section{References:}

[1] W. Chao et al, Nature 435 (2005) p. 1210-1213.

[2] A. Tkachuk et al, Z. Kristallogr. 222 (2007) p. 650-655.

[3] M. Dierick et al, Nucl. Instrum. Methods Phys. Res. B. 324 (2014) p. 35-40.

[4] M. Müller et al, PNAS 114 (2017) p. 12378-12383.

[5] M. Busse et al, PNAS 115 (2018) p. 2293-2298.

[6] P. Kraft et al, J. Synchrotron Radiat. 16 (2009) p. 368-375.

[7] B. Metscher, Cold Spring Harb Protoc. 12 (2011) p. 1462-1471.

[8] 'Zebrafish embryo' by Annie Cavanagh. Credit: Annie Cavanagh. CC BY-NC, https://blog.wellcome.ac.uk/2013/07/19/wellcome-image-of-the-month-zebrafish-embryo/, accessed March 20, 2018, license: https://creativecommons.org/licenses/by-nc/4.0

[9] We thank the very helpful team at Excillum AB, Sweden. The work was supported by the German Research Foundation (DFG) Cluster of Excellence Munich-Center for Advanced Photonics, the DFG Gottfried Wilhelm Leibniz program, and the Technical University of Munich Institute for Advanced Study, funded by the German Excellence Initiative. 